\title{
Doppler Parameter Estimation Based on Fractional Correlation in Alpha-Stable Distribution Noise Environment
}

\author{
$\mathrm{Li} \mathrm{Li} i^{1, a}$ * \\ ${ }^{1}$ College of Information Engineering, Dalian University, Dalian 116622, Chin \\ affsimple@163.com
}

Keywords: Fractional correlation; Fractional lower order statistics; Doppler frequency; $\alpha$-stable distribution

\begin{abstract}
This paper takes the -stable distribution as the noise model and works on the parameter estimation problem in the impulsive noise environment. This paper presents a signal model and a novel method for parameter estimation in the impulsive noise environment. Firstly, a signal array model is constructed based on the $\alpha$-stable distribution model. Secondly, Doppler parameters are jointly estimated by searching the optimal rotation angle to meet concentrated-energy of the FLOS-FC. Simulation results are presented to verity the effectiveness of the proposed method.
\end{abstract}

\section{Introduction}

The fractional Fourier transform (FRFT), which is a generalization of the classical Fourier transform, was introduced a number of years ago in the mathematics literature but appears to have remained largely unknown to the signal processing community, to which it may, however, be potentially useful. The FRFT depends on a parameter and can be interpreted as a rotation by an angle in the time-frequency plane.

Target parameter estimation is an important aspect in radar signal processing. Most existing algorithms present that Doppler frequency is time-invariant as shown in [1-3]. In fact, the received signals contain time-variant Doppler frequency due to the three dimension motion state of these targets. In this case, these existing methods can not effectively solve this problem and provide an optimal solution[5-10].

Furthermore, in most parameter estimation methods for array signal processing, additive noise is assumed to be Gaussian, which has finite second-order statistics. In some scenarios, it is inappropriate to model the noise as Gaussian noise. To overcome these drawbacks, this paper presents a novel method, combining the fractional lower order statistics and fractional correlation, to suppress the impulse noise and estimate parameters in the impulsive noise environment.

\section{The Signal Model}

In radar or a sonar system, the received signal, in comparision with the transmitted waveform, often contains Doppler frequency. We assume that the transmit antennas emit orthogonal waveforms $x(t)$. Let $x(t)$ be the transmitted signal having a constant propagation velocity. The received signals contain Doppler frequency with time-variant. In general, the echo signal $y(t)$ can be expressed as

$$
y(t)=\sum_{l=1}^{L} \sigma_{l} x(t) \exp \left(j 2 \pi\left(f_{l} t+\mu_{l} t^{2} / 2\right)\right)+w(t)
$$

where $\sigma_{l}$ denotes the radar cross-section. $f_{l}$ and $\mu_{l}$ denote the initial Doppler frequency (IDF) and Doppler frequency rate (DFR). $L$ is the number of multiple paths. The noise $w(t)$ is a sequence of i.i.d isotropic complex $S \alpha S$ random variable with $1<\alpha \leq 2$.

\section{Theory of Parameter Estimation based on the FLOS-FC}


FRFT. The FRFT is a generalization of the FT, and can be interpreted as a rotation of the signal to any angles in the time-frequency plane ${ }^{[4]}$. The continuous FRFT of a signal $f(t)$ with angle $\rho$ is defined as

$$
F(\rho, m)=F^{\rho}[f(t)](m)=\int_{-\infty}^{+\infty} f(t) K_{\rho}(t, m) d t
$$

where $F^{\rho}$ denotes the FRFT operator and $K_{\rho}(t, m)$ is the kernel function of the fractional Fourier transform. $K_{\rho}(t, m)$ can be expressed as

$$
K_{\rho}(t, m)= \begin{cases}\sqrt{(1-j \cot \rho)} \exp \left(j \pi\left(t^{2} \cot \rho-2 m t \csc \rho+m^{2} \cot \rho\right)\right), \rho \neq n \pi \\ \delta(t-m), & \rho=2 n \pi \\ \delta(t+m), & \rho=(2 n+1) \pi\end{cases}
$$

where $\rho$ and $m$ are the rotation angle and the frequency in FRFT domain, respectively.

Fractional Correlation Function. Assume that the signal $s(t)$ is modeled as

$$
s(t)=b_{0} \exp \left(j 2 \pi\left(a_{1} t+a_{2} t^{2} / 2\right)\right)
$$

where $b_{0}$ is the signal amplitude, $a_{1}$ is the initial frequency and $a_{2}$ is frequency rate.

Fractional correlation function $\hat{R}_{s s}^{\rho}(\tau)$ of the signal $s(t)$ is defined by

$$
\hat{R}_{s s}^{\rho}(\tau)=\lim _{T \rightarrow \infty} \frac{1}{2 T} \int_{-T}^{+T} R_{s s}(t+\tau, t) \exp (j t \tau \cot \rho) d t
$$

where $R_{s s}(t+\tau, t)$ is the correlation function of the signal $s(t),{ }^{\tau}$ denotes the time delay, and $\rho$ is the rotation angle in FRFT domain.

Fractional Correlation Function Based on the Fractional Lower-Order Statistics. $\alpha$-stable distribution has heavier tails in its probality function than the normal distribution, possess finite $p$ th-order moments only for $p<\alpha$, and is more appropriate than the usual Gaussian assumption for the noise with extreme outliers[8-9]. This paper combines the fractional lower order statistics and fractional correlation function for suppressing the impulse noise and estimating parameters.

Fractional correlation function based on the fractional lower order statistics (FLOS-FC) $\hat{R}_{s s}^{(p)}(\tau)$ of the signal $s(t)$ is defined by

$$
\hat{R}_{s s}^{(p)}(\tau)=\lim _{T \rightarrow \infty} \frac{1}{2 T} \int_{-T}^{+T} R_{s s}^{(p)}(t+\tau, t) \exp (j t \tau \cot \rho) d t
$$

where $p$ is the order of the fractional lower order, and $1<p<\alpha \leq 2$. $R_{s s}^{(p)}(t+\tau, t)=E\left\{s(t+\tau)[s(t)]^{<p-1>}\right\}$ denotes the fractional lower order correlation of the signal $s(t)$.

\section{Joint Doppler Frequency Parameter Estimation Based on FLOS-FC}

According to (1) and (6), the FLOS-FC of $y(t)$ can be written as

$$
\hat{R}_{y y}^{(p)}(\tau)=\lim _{T \rightarrow \infty} \frac{1}{2 T} \int_{-T}^{+T} R_{y y}^{(p)}(t+\tau, t) \exp (j t \tau \cot \alpha) d t
$$

where $R_{y y}^{(p)}(t+\tau, t)=E\left\{y(t+\tau)[y(t)]^{<p-1>}\right\}$.

When $\cot \rho_{l}=-2 \pi \mu_{l}, \hat{R}_{y y}^{(p)}(\tau)$ has the best energy-concentrated property. Therefore, we can obtain the following expression as

$$
\left\{\begin{array}{l}
\hat{\mu}_{l}=-\cot \rho_{l} /(2 \pi) \\
\hat{f}_{l}=\arg \max _{f}\left\{\tilde{R}_{y_{1}}^{p}(u)\right\}
\end{array}\right.
$$

where $\tilde{R}_{y_{1}}^{(p)}(u)$ is the Fourier transform of $R_{y_{1}}^{(p)}(t)$, and $R_{y_{1}}^{(p)}(t)$ is the fractional autocorrelation function of $y_{1}(t)=y_{q, n, l}(t) \cdot \exp \left(-\mathrm{j} 2 \pi\left(\hat{\mu}_{l} / 2\right) t^{2}\right)$. 


\section{Test Results}

In order to study the robustness of the propose method, complex isotropic symmetric $\alpha$-stable $(S \alpha S)$ noise $^{[8]}$ model is considered. We describe the signal-to-noise condition of $S \alpha S$ using the generalized signal-to-noise $\left(\operatorname{GSNR}=10 \lg \left(\sigma_{\mathrm{s}}^{2} / \gamma\right)\right)$, where, $\sigma_{\mathrm{s}}^{2}$ is the signal power.

Supposed Doppler parameters are $f_{1}=8, \mu_{1}=1.2, f_{2}=10, \mu_{2}=2$. respectively. The number of snapshots is 1000 . The number of Monte Carlo iterations is 500 in all simulations.

Simulation 1: Generalized signal-noise-ratio GSNR

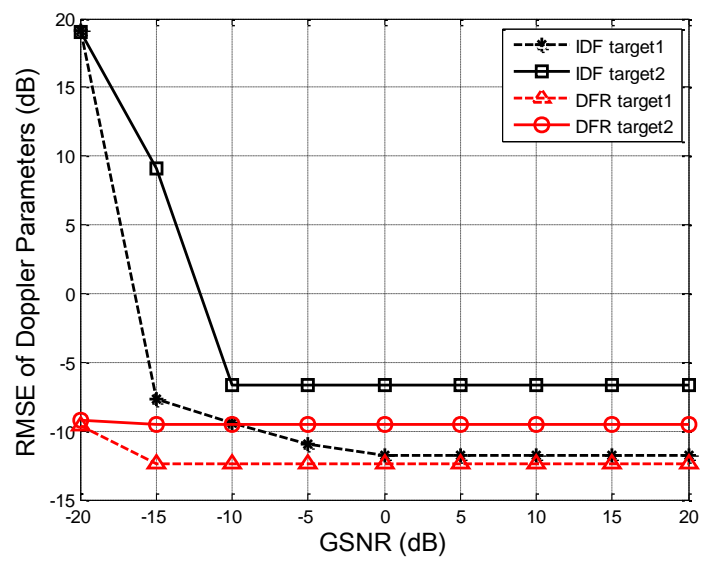

(a)

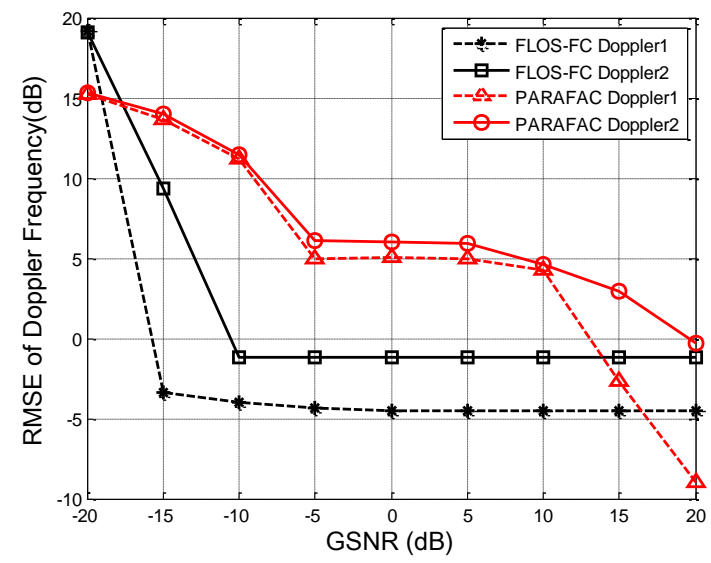

(b)

Figure 1. Root mean square error versus GSNR

In this simulation, the noise is modeled as $\alpha$-stable noise with $\alpha=1.4$ and the fractional lower-order moment is set as $p=1.2$. Fig. 1 (a) depicts the RMSE of Doppler parameters. Fig. 1 (b) shows Doppler frequency estimation performance of two algorithms for various values of GSNR . From Fig. 1, we can find that the estimation accuracy of the proposed method is higher than those of PARAFAC method.

Simulation 2: Characteristic exponent $\alpha$

In this simulation, The generalized signal to noise ratio is set as GSNR $=15 \mathrm{~dB}$, and the fractional lower-order moment is set as $p=1.2$. Fig. 2 shows the RMSE of the three algorithms for various values of characteristic exponent $\alpha$.

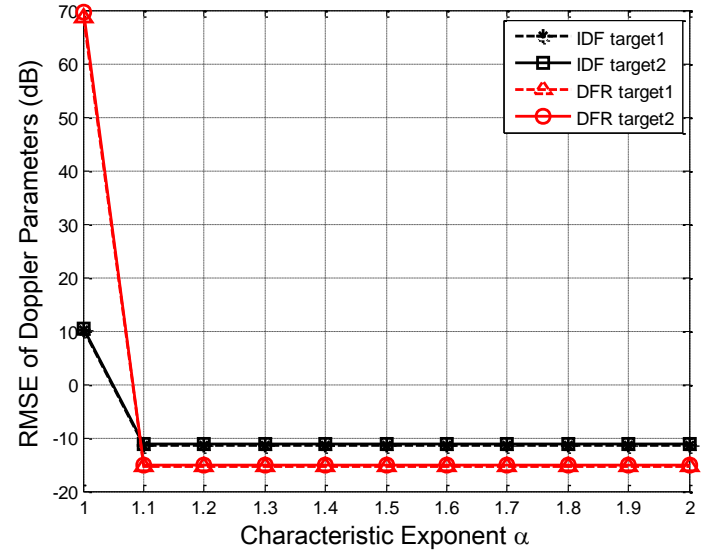

(a)

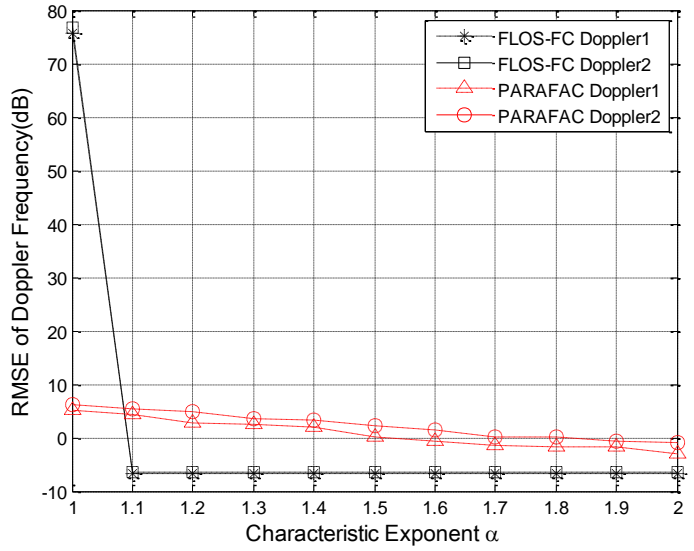

(b)

Figure 2. Root mean square error versus $\alpha$

From Fig. 2, we find that the proposed algorithm shows a significant performance improvement over the PARAFAC method in term of RMSE for values of $\alpha$ in the range $(1,2]$ in impulsive noise environments modeled under the stable law. 


\section{Conclusions}

In this paper, we proposed a novel method for estimating unknown targets parameters in impulse noise environment. Firstly, we present a signal model under Alpha-stable distribution noise in bistatic MIMO radar system. Secondly, Doppler parameters are estimated by the FLOS-FC algorithm. Simulation results demonstrate that the proposed method still has good performance when poor SNR condition exists.

\section{Acknowledgments}

This work was partly supported by the National Science Foundation of China under Grants 61401055.

\section{References}

[1] C.Y. Chen and P.V. Palghat, MIMO radar space-time adaptive processing using prolate spheroidal wave functions, IEEE Transactions on Signal Processing, 56(2008) 623-635.

[2] C.C. Li, et al, An ESPRIT-like algorithm for coherent DOA estimation based on data matrix decomposition in MIMO radar, Signal Processing, 91(2011) 1803-1811.

[3] M.L. Bencheikh, Y.D. Wang and H.Y. He, Polynomial root finding technique for joint DOA DOD estimation in bistatic MIMO radar, Signal Processing, 89(2010) 2723-2730.

[4] R. Tao, F. Zhang, Y. Wang, Fractional Power Spectrum, IEEE Trans. Signal Processing, 56(2008) 4199-4206.

[5] H. Lu, et al, A novel method for target localization and doppler frequency estimation in bistatic MIMO radar, Journal of Electronics and Information Technology, 32(2010) 2167- 2171.

[6] X.F. Zhang, et al, Direction of departure (DOD) and direction of arrival (DOA) estimation in MIMO radar with reduced-dimension MUSIC, IEEE Communications Letters, 14(2010) 1161-1163.

[7] J.Y. Zhang, Z.D. Zheng, and X.B. Li, An algorithm for DOD-DOA and Doppler frequency jointly estimating of bistatic MIMO radar, Journal of Electronics and Information Technology, 32(2010): 1843-1848.

[8] C.L.Nikias and S. Min, Signal processing with alpha-stable distributions and applications. Wiley-Interscience, 1995.

[9] L.B. Almeida, The fractional Fourier Transform and Time-Frequency Representations, IEEE Trans. Signal Processing, 42(1994):3084-3091.

[10] J.T. Wang, et al. Subspace-based DOA Estimation in Impulsive Noise Environments for MIMO Radars, Journal of Astronautics, 30(2009) 1653-1657. 